\title{
Auricular Acupuncture for Preoperative Anxiety - Protocol of Systematic Review and Meta-Analysis of Randomized Controlled Clinical Trials.
}

Joanna Dietzel ( $\sim$ joanna.dietzel@charite.de)

Charite Universitatsmedizin Berlin https://orcid.org/0000-0003-4739-4230

Mike Cummings

British Medical Acupuncture Society

Kevin Hua

Charite Universitatsmedizin Berlin

Klaus Hahnenkamp

Universitatsmedizin Greifswald

Benno Brinkhaus

Charite Universitatsmedizin Berlin

Taras Usichenko

Universitatsmedizin Greifswald

\section{Protocol}

Keywords: auricular acupuncture, preoperative anxiety, protocol, randomized controlled trials, systematic review, meta-analysis

Posted Date: August 7th, 2020

DOl: https://doi.org/10.21203/rs.3.rs-54205/v1

License: (c) (i) This work is licensed under a Creative Commons Attribution 4.0 International License.

Read Full License 


\section{Abstract}

Background: Preoperative anxiety causes a profound psychological and physiological reactions, that may lead to a worse postoperative recovery, higher intensity of acute and persistent postsurgical pain and impaired quality of life in the postoperative period. Previous randomized controlled trials (RCTs) suggest that auricular acupuncture (AA) is safe and effective in treatment of preoperative anxiety; a systematic evidence on this topic is missing. Therefore, this protocol was developed following the PRISMA guidelines to adequately evaluate the existing literature regarding the value of $A A$ for the reduction of anxiety in patients in a preoperative setting, compared to other forms of acupuncture or pharmacological or no control interventions and measured with questionnaires regarding anxiety and fear.

Methods and analysis: The following databases will be searched: MEDLINE (PubMed), EMBASE, Cochrane Central Register of Controlled Trials (CENTRAL), ISI Web of Science, Scopus Database and Google search. RCTs will be included if an abstract is available in English. Data collection and analysis will be conducted by 2 reviewers independently. Quality and risk assessment of included studies will be done using the Cochrane 5.1.0 handbook criteria and meta-analysis of effectiveness and symptom scores will be conducted using the statistical software RevMan V.5.3.

Ethics and dissemination: This systematic review will evaluate the efficacy and safety of AA for preoperative anxiety. Since all data used in this systematic review and meta-analysis have been published, this review does not require ethical approval. The results may be published in a peer-reviewed journal or be presented in relevant conferences.

Registration number: PROSPERO ID CRD42020184795

\section{Introduction}

\subsection{Background}

Preoperative anxiety is the most frequent burden affecting patients before various surgical procedures (1). Facing the need for surgery and hospitalization, patients experience fear, anxiety, uncertainty, loss of control and decreased self-esteem (2). More than $90 \%$ of adult patients, scheduled for elective surgery, developed preoperative anxiety, and $40.5 \%$ reported severe anxiety (3). Although the preoperative anxiety is a kind of situational anxiety, which terminates itself when the underlying condition (surgery) is over, it causes a profound psychological and physiological response, that may be associated with a worse postoperative recovery, higher intensity of acute and chronic persistent postsurgical pain and greater anesthetic requirement as well as with impaired quality of life in the postoperative period (4-8).

A large variety of approaches is used to treat preoperative anxiety including both psychological and pharmacological interventions; however, none of them seem to be ideal in providing effective, safe and low-cost therapy $(9-11)$. 
Auricular stimulation (including acupuncture and comparable techniques such as electroacupuncture and acupressure) is a complementary medicine method, based on stimulation of cranial nerves, and this has already been used to treat situational anxiety in experimental and clinical conditions (12). In randomised controlled trials (RCTs) for the treatment of preoperative anxiety, auricular stimulation was superior to an array of control procedures, including placebo and sham interventions, and was similar in effect to premedication with benzodiazepines in patients scheduled for surgery under general anesthesia. (1317). Moreover, this method was associated with fewer side effects compared with benzodiazepines, as well as with a diminished stress reaction, measured as a reduced response of the autonomic nervous system $(12,16,17)$.

The potential mechanism of auricular stimulation is attributed to the neuroanatomical conditions of external auricle. It is presumed, that auricular stimulation exerts its anxiolytic effects via the involvement of cranial nerves (18), which leads to the modulation of the brain areas involved in the stress response, including the limbic system, locus coeruleus and hypothalamus (19-21).

Although the majority of RCTs on auricular stimulation for preoperative anxiety were in favour of this technique, these clinical investigations demonstrated a heterogeneity in regard to surgical procedures, control conditions and effect size, thus making it difficult to draw any definitive recommendations. It seems possible that auricular stimulation might serve as an effective replacement for insufficient conventional pharmacological premedication $(9,22)$, thus more accurate estimation of the efficacy and safety of this complementary medicine intervention is needed. Therefore, the present systematic review including meta-analysis of RCTs will be performed to evaluate the effect size of auricular stimulation on preoperative anxiety applied alone or in addition to standard care in comparison with various control conditions. The review will also try to identify the factors that may influence the effects of this intervention.

\section{Methods}

This systematic review protocol has been registered on PROSPERO ID CRD42020184795. The protocol follows the Cochrane Handbook for Systematic Reviews of Interventions. We will describe any changes in our full review if needed. Since all data used in this systematic review and meta-analysis have been published, this review does not require ethical approval.

\subsection{Eligibility Criteria for including studies in the review 2.1.1. Types of studies}

Only randomized controlled trials (RCTs) in European languages will be included. Results from quasi RCT will be discussed if little evidence is available, but they will not be part of the analysis. The funding source will be registered.

\subsubsection{Types of participants}


No restrictions on study populations will be made, as long as they are described as patients, undergoing surgical procedures, including all medical interventions requiring intra-procedural sedation or analgesia. There will be no restrictions regarding the age, gender or ethnicity of participants.

\subsubsection{Types of interventions/comparators}

This review will include all studies, where auricular stimulation or related interventions (auricular acupuncture, auricular acupressure, auricular electroacupuncture, etc.) applied alone or in addition to routine care will be compared with a variety of control conditions, such as: sham acupuncture, acupressure, placebo, routine care, various cognitive-behavioral therapies (CBTs) such as relaxation techniques, music therapy, hypnosis, etc.

\subsubsection{Types of outcome measures}

\subsubsection{Primary outcomes}

The primary outcome for this review will be an intensity of preoperative anxiety, measured using patientreported psychophysical anxiety scales, such as the State Trait Anxiety Inventory (STAI), Anxiety Visual Analogue Scale-100 (VAS-100), the Amsterdam Preoperative Anxiety and Information Scale (APAIS), SelfRating Anxiety Scale (SAS), extensively described elsewhere (23).

\subsubsection{Secondary outcomes}

Secondary outcomes will include physiological parameters describing the response of the autonomic nervous system (e.g. heart rate, blood pressure, respiratory rate, sweating reaction); the preoperative requirement of anxiolytic medication; the intraoperative requirement of anaesthetic and analgesic medication; the intensity of postoperative pain; the postoperative requirement for analgesic medication and patient satisfaction with the treatment of preoperative anxiety.

\subsubsection{Safety of intervention.}

Adverse event and serious adverse events reporting will be analysed, including events such as pain, inflammation and infection at the sites of auricular stimulation, and vasovagal reactions during the auricular interventions.

\subsection{Search methods for identification of studies}

\subsubsection{Electronic searches.}

The search will be done across the following electronic databases and registers, from their inception till June 2020: MEDLINE (PubMed), EMBASE, Cochrane Central Register of Controlled Trials (CENTRAL), ISI Web of Science, Scopus Database, Google search. The search terms will include: auricular, acupuncture, acupressure, preoperative anxiety, randomized clinical trials. The complete search strategy is listed in Table 1. 
Table 1

Search strategy used in MEDLINE database

\begin{tabular}{|ll|}
\hline $\mathbf{N}$ & Search item [title/ abstract] \\
\hline 1 & Randomized controlled trial \\
\hline 2 & Controlled clinical trial \\
\hline 4 & Randomized \\
\hline 5 & Randomly \\
\hline 6 & Trial \\
\hline 7 & OR \#1-6 \\
\hline 8 & Anxiety \\
\hline 9 & Fear \\
\hline 11 & Preoperative \\
\hline 12 & Surgical \\
\hline 14 & Antervention \\
\hline 15 & OR \#7-14 \\
\hline 16 & Auricular acupuncture \\
\hline 17 & Auricular \\
\hline 18 & Ear \\
\hline 19 & Acupressure \\
\hline 20 & Electro-acupuncture \\
\hline This search & strategy will be modified as required for other electronic databases. \\
\hline
\end{tabular}

\subsection{Data extraction and management 2.3.1 Study identification}

Two researchers will screen the titles and abstracts of articles found in the search, and discard trials that are not eligible. They will assess independently whether the trials meet the inclusion criteria, with disagreements to be resolved by discussion with the third author. When articles contain insufficient information to make a decision about eligibility, one of the researchers will attempt to contact authors of the original reports to obtain further details via email. The details of data search and management are 
given as Fig. 1. A new database for each of the two researchers will be set up to organize the data of the literature search.

\subsubsection{Data extraction.}

Following the selection for inclusion, two researchers will independently extract data according to the standardized form designed by the review group (Table 2). A third researcher will check for accuracy and enter data into Review Manager software (RevMan 5.3. 2011).

Table 2

Content of data extraction

\begin{tabular}{|c|c|c|}
\hline $\mathbf{N}$ & Categories & Items extracted \\
\hline 1 & $\begin{array}{l}\text { General } \\
\text { information }\end{array}$ & $\begin{array}{l}\text { Author, year of publication, title, journal (title, volume, pages), country, language } \\
\text { of publication }\end{array}$ \\
\hline 2 & $\begin{array}{l}\text { Research } \\
\text { method }\end{array}$ & Random allocation, allocation concealment, blinding, baseline level \\
\hline 3 & Participants & $\begin{array}{l}\text { Total sample size, number in experimental group, number in control group, } \\
\text { gender, age, ethnicity, type of surgery, setting }\end{array}$ \\
\hline 4 & Intervention & $\begin{array}{l}\text { Type of intervention (auricular acupuncture, auricular acupressure, auricular } \\
\text { electro-acupuncture, etc.), selection of auricular sites/auricular acupuncture } \\
\text { points, selected for stimulation, type of device/needles, used for auricular } \\
\text { stimulation, length of auricular stimulation, control condition }\end{array}$ \\
\hline 5 & $\begin{array}{l}\text { Outcome } \\
\text { parameter }\end{array}$ & $\begin{array}{l}\text { Levels of preoperative anxiety (taken using questionnaires and psychophysical } \\
\text { scales), physiological parameters (heart rate, blood pressure, respiratory rate, } \\
\text { sweating reaction, etc.), preoperative requirement of anxiolytic medication, } \\
\text { intraoperative requirement of anaesthetic and analgesic medication, the intensity } \\
\text { of postoperative pain, postoperative requirement of analgesic medication, patient } \\
\text { satisfaction with the treatment of preoperative anxiety }\end{array}$ \\
\hline
\end{tabular}

\subsubsection{Assessment of risk of bias in included studies}

Two researchers will assess all included trials for risk of bias, blind to each other's assessments. Random sequence generation, allocation concealment, blinding of participants and personnel, incomplete outcome data, selective reporting and other potential sources of bias will be evaluated regarding low, high and unclear risk of bias according to Cochrane Collaboration assessment tool. Any disagreements will be resolved by discussion or by involving a third researcher to adjudicate.

\subsubsection{Measures of treatment effects}

Since all outcome measures of this review represent continuous data, they will be presented as mean differences with 95\% confidence intervals (CI), or as standardized mean differences (SMD).

\subsubsection{Dealing with missing data.}


All outcomes will be analyzed on an intention-to-treat basis. Corresponding authors from the trials with incomplete or insufficient data will be contacted via email to complete the data. Trials with greater than $20 \%$ of missing data will be excluded from the analysis.

\subsubsection{Assessment of heterogeneity}

Statistical heterogeneity will be assessed in each meta-analysis using the $T^{2}, R$ and Chi ${ }^{2}$ statistics calculated by RevMan software. Heterogeneity will be regarded as substantial if $T^{2}$ is greater than zero and either $P$ is greater than $50 \%$ or there is a low $P$ value (less than 0.10 ) in the Chi ${ }^{2}$ test for heterogeneity.

\subsubsection{Assessment of reporting biases}

If the meta-analysis includes more than 10 investigations, reporting biases will be studied using a funnel plot with asymmetry testing.

\subsection{Data synthesis}

Statistical analysis will be carried out using the RevMan software. Fixed-effect meta-analysis for combining data including primary outcome (anxiety scales) will be performed to estimate the treatment effect using SMD and 95\% Cl. In case of substantial clinical or statistical heterogeneity, a random-effects (RE) meta-analysis will be done to yield an overall summary. If RE analyses will be necessary, their results will be presented as the average treatment effect with its $95 \%$ confidence interval, and the estimates of $T^{2}$ and $R$.

\subsubsection{Subgroup analysis and investigation of heterogeneity}

To assess potential heterogeneity, subgroup analyses will be performed including following comparisons: adult versus pediatric patients; female versus male patients; emergency surgery versus elective surgery; inpatient versus outpatient surgery. Differences between subgroups will be assessed by interaction tests for fixed-effect inverse variance meta-analyses. For fixed-effect meta-analyses and RE using methods other than inverse variance, the comparison of subgroups' confidence intervals will be used: nonoverlapping confidence intervals indicate a statistically significant difference in treatment effect between the subgroups.

\subsubsection{Sensitivity analysis}

Where subgroup analysis fails to explain the heterogeneity, data analysis using the RE model will be used. A priori, sensitivity analyses on results will be done to look at the possible contribution of differences in methodological quality, comparing trials with a low risk of bias to all trials.

\subsubsection{Quality of outcome evidence}

The quality of outcome evidence will be summarized using the Grading of Recommendations Assessment, Development and Evaluation (GRADE) approach. Each grade of evidence will be rated as: high, moderate, low or very low. 


\subsection{Discussion}

Although almost $50 \%$ of adult patients, scheduled for elective surgery, suffer from preoperative anxiety (3), there is no ideal method to treat this kind of situational anxiety in clinical conditions so far. Pharmacological premedication is convenient in preoperative setting, however it seems to be less effective than previously suggested, if compared with placebo in trials with rigorous designs $(9,22)$. Psychological (cognitive-behavioral) approaches seem to be effective and lack dangerous side effects, however they are too time-consuming in their execution and thus are seldom used in routine clinical practice (24).

An array of data suggest that auricular stimulation might become such an effective, safe and easy-toperform treatment for preoperative anxiety in adults, scheduled for elective surgery and painful procedures with sedation (12-17). Despite these promising results from clinical trials on treatment of preoperative anxiety using auricular stimulation, supported by neurophysiological explanation of its potential mechanisms (25), the systematic evaluation of evidence for treatment of anxiety using auricular acupuncture is not available.

This review and meta-analysis will fill this gap, analyzing the RCTs based on this protocol, which was designed according to the PRISMA statement. The review will calculate and summarise the data on efficacy and safety of auricular stimulation in the treatment of preoperative anxiety in adult patients scheduled to elective surgery.

The results of this systematic review may be biased, since only the trials described in European languages will be considered, excluding the full format papers in native languages from the countries of the Far East, where auricular stimulation is widely used in traditional medicine (26). Furthermore, the trials using transauricular vagal nerve stimulation (TaVNS) are considered to be beyond the scope of this review, despite the number of such trials growing rapidly in last two decades (27).

In summary, this systematic review will evaluate the existing evidence on the treatment of preoperative anxiety using auricular acupuncture and related procedures. The scheduled meta-analysis will estimate the effect of auricular stimulation on several perioperative parameters, that are known to be influenced by preoperative anxiety. The results of this review will provide the basis for better understanding of auricular acupuncture in treatment of perioperative anxiety and will yield the evidence for implementation of this method in clinical practice.

\section{Registry}

This protocol has been registered in the PROSPERO registry (International prospective register of systematic reviews) under the ID CRD42020184795.

\section{Abbreviations}


RCT: randomized controlled trial, AA: Auricular acupuncture, STAl: State Trait Anxiety Inventory, VAS-100: Anxiety Visual Analogue Scale-100, APAIS: Amsterdam Preoperative Anxiety and Information Scale, SAS: Self-Rating Anxiety Scale, CBT: cognitive-behavioral therapies, Cl: confidence intervals, SMD: standardized mean differences, RE: random-effects, GRADE: Grading of Recommendations Assessment, Development and Evaluation, TaVNS: using transauricular vagal nerve stimulation.

\section{Declarations}

Ethics approval: not applicable

Consent for publication: not applicable

Availability of data and materials: The datasets used and/or analysed during the current study are available from the corresponding author on reasonable request.

Competing interests: The authors declare that they have no competing interests.

Funding: Joanna Dietzel received a research grant from the Karl and Veronica Carstens foundation for conducting several clinical studies and a systematic review with meta-analysis. The foundation had no role in the development of the protocol. Karl and Veronica Carstens foundation, Am Deimelsberg 36, 45276 Essen, Germany. Klaus Hahnenkamp and Benno Brinkhaus provide publication and open access fees.

Author contributions: Data collection: Joanna Dietzel, Kevin Hua, Taras Usichenko

Formal analysis: Mike Cummings Supervision: Mike Cummings, Klaus Hahnenkamp

Writing-original draft: Joanna Dietzel, Taras Usichenko, Benno Brinkhaus Writing - review \& editing: Joanna Dietzel, Taras Usichenko, Mike Cummings

\section{References}

1. Walker EM, Bell M, Cook TM, Grocott MP, Moonesinghe SR. Patient reported outcome of adult perioperative anaesthesia in the United Kingdom: a crosssectional observational study. $\mathrm{Br} \mathrm{J}$ Anaesth. 2016;117:758-66.

2. Breemhaar B, van den Borne HW, Mullen PD. Inadequacies of surgical patient education. Patient Educ Couns. 1996;28:31\&\#8211.

3. Eberhart L, Aust H, Schuster M, Sturm T, Gehling M, Euteneuer F, Rüsch D. Preoperative anxiety in adults - a cross-sectional study on specific fears and risk factors. BMC Psychiatry. 2020;20:140.

4. Maranets_I Kain_ZN. Preoperative anxiety and intraoperative anesthetic requirements. Anesth Analg. 1999;89:1346-51. 
5. Kain_ZN Sevarino_F, Alexander_GM, Pincus_S Mayes_LC. Preoperative anxiety and postoperative pain in women undergoing hysterectomy. A repeated-measures design. J Psychosom Res. 2000;49:417-22.

6. Scott_A. Managing anxiety in ICU patients: the role of preoperative information provision. Nurs Crit Care. 2004;9:72-9.

7. Theunissen M, Peters ML, Bruce J, Gramke HF, Marcus MA. Preoperative anxiety and catastrophizing: a systematic review and meta-analysis of the association with chronic postsurgical pain. Clin J Pain. 2012;28:819-41.

8. Ali A, Altun D, Oguz BH, Ilhan M, Demircan F, Koltka K. The effect of preoperative anxiety on postoperative analgesia and anesthesia recovery in patients undergoing laparascopic cholecystectomy. J Anesth. 2014;28:222-7.

9. Maurice-Szamburski A, Auquier P, Viarre-Oreal V, Cuvillon P, Carles M, Ripart J, Honore S, Triglia T, Loundou A, Leone M, Bruder N. PremedX Study Investigators. Effect of sedative premedication on patient experience after general anesthesia: a randomized clinical trial. JAMA. 2015;313:916-25.

10. Powell R, Scott NW, Manyande A, Bruce J, Vögele C, Byrne-Davis LM, Unsworth M, Osmer C, Johnston M. Psychological preparation and postoperative outcomes for adults undergoing surgery under general anaesthesia. Cochrane Database Syst Rev. 2016;5:CD008646.

11. Kim J, Chiesa N, Raazi M, Wright KD. A systematic review of technology-based preoperative preparation interventions for child and parent anxiety. Can J Anaesth. 2019;66:966-86.

12. Pilkington K, Kirkwood G, Rampes H, Cummings M, Richardson J. Acupuncture for anxiety and anxiety disorders-a systematic literature review. Acupunct Med. 2007;25:1-10.

13. Wang SM, Maranets I, Weinberg ME, Caldwell-Andrews AA, Kain ZN. Parental auricular acupuncture as an adjunct for parental presence during induction of anesthesia. Anesthesiology. 2004;100:1399404.

14. Michalek-Sauberer, et al. Auricular acupuncture effectively reduces state anxiety before dental treatment-a randomised controlled trial. Clin Oral Investig. 2012;16:1517-22.

15. Luo L, Dai Q, Mo Y, Yan Y, Zhuang X, Hunag L, et al. The effect of auricular acupressure on preoperative anxiety in patients undergoing gynecological surgery. Int J Clin Exp Med. 2016;9:406570.

16. Lewis GBH, Litt M. An alternative approach to premedication: comparing diazepam with auriculotherapy and a relaxation method. Am J Acupunct. 1987;15:205-11.

17. Karst M, Winterhalter M, Münte S, Francki B, Hondronikos A, Eckardt A, Hoy L, Buhck H, Bernateck M, Fink M. Auricular acupuncture for dental anxiety: a randomized controlled trial. Anesth Analg. 2007;104:295-300.

18. Peuker ET, Filler TJ. The nerve supply of the human auricle. Clin Anat. 2002;15:35-7.

19. Frangos E, Ellrich J, Komisaruk BR. Non-invasive Access to the Vagus Nerve Central Projections via Electrical Stimulation of the External Ear: fMRI Evidence in Humans. Brain Stimul. 2015;8:624-36. 
20. Chen J, Barrett DW, He Y, Gonzalez-Lima F. Anxiolytic-like behavioural effects of head electroacupuncture in rats susceptible to stress. Acupunct Med. 2016;34:235-40.

21. Qu F, Zhang D, Chen LT, Wang FF, Pan JX, Zhu YM, Ma CM, Huang YT, Ye XQ, Sun SJ, Zheng WJ, Zhang RJ, Xu J, Xing LF, Huang HF. Auricular acupressure reduces anxiety levels and improves outcomes of in vitro fertilization: a prospective, randomized and controlled study. Sci Rep. 2014;4:5028.

22. Walker KJ, Smith AF. Premedication for anxiety in adult day surgery. Cochrane Database Syst Rev. 2009;4:CD002192.

23. Zemła AJ, Nowicka-Sauer K, Jarmoszewicz K, Wera K, Batkiewicz S, Pietrzykowska M. Measures of preoperative anxiety. Anaesthesiol Intensive Ther. 2019;51:64-9.

24. Wilson CJ, Mitchelson AJ, Tzeng TH, El-Othmani MM, Saleh J, Vasdev S, LaMontagne HJ, Saleh KJ. Caring for the surgically anxious patient: a review of the interventions and a guide to optimizing surgical outcomes. Am J Surg. 2016;212:151-9.

25. Usichenko T, Hacker $\mathrm{H}$, Lotze M. Transcutaneous auricular vagal nerve stimulation (taVNS) might be a mechanism behind the analgesic effects of auricular acupuncture. Brain Stimul. 2017;10:1042-4.

26. Kim YS, Jun H, Chae Y, Park HJ, Kim BH, Chang IM, Kang SK, Lee HJ. The practice of Korean medicine: an overview of clinical trials in acupuncture. Evid Based Complement Alternat Med. 2005;2:325-52.

27. Carreno FR, Frazer A. The Allure of Transcutaneous Vagus Nerve Stimulation as a Novel Therapeutic Modality. Biol Psychiatry. 2016;79:260-1.

\section{Figures}




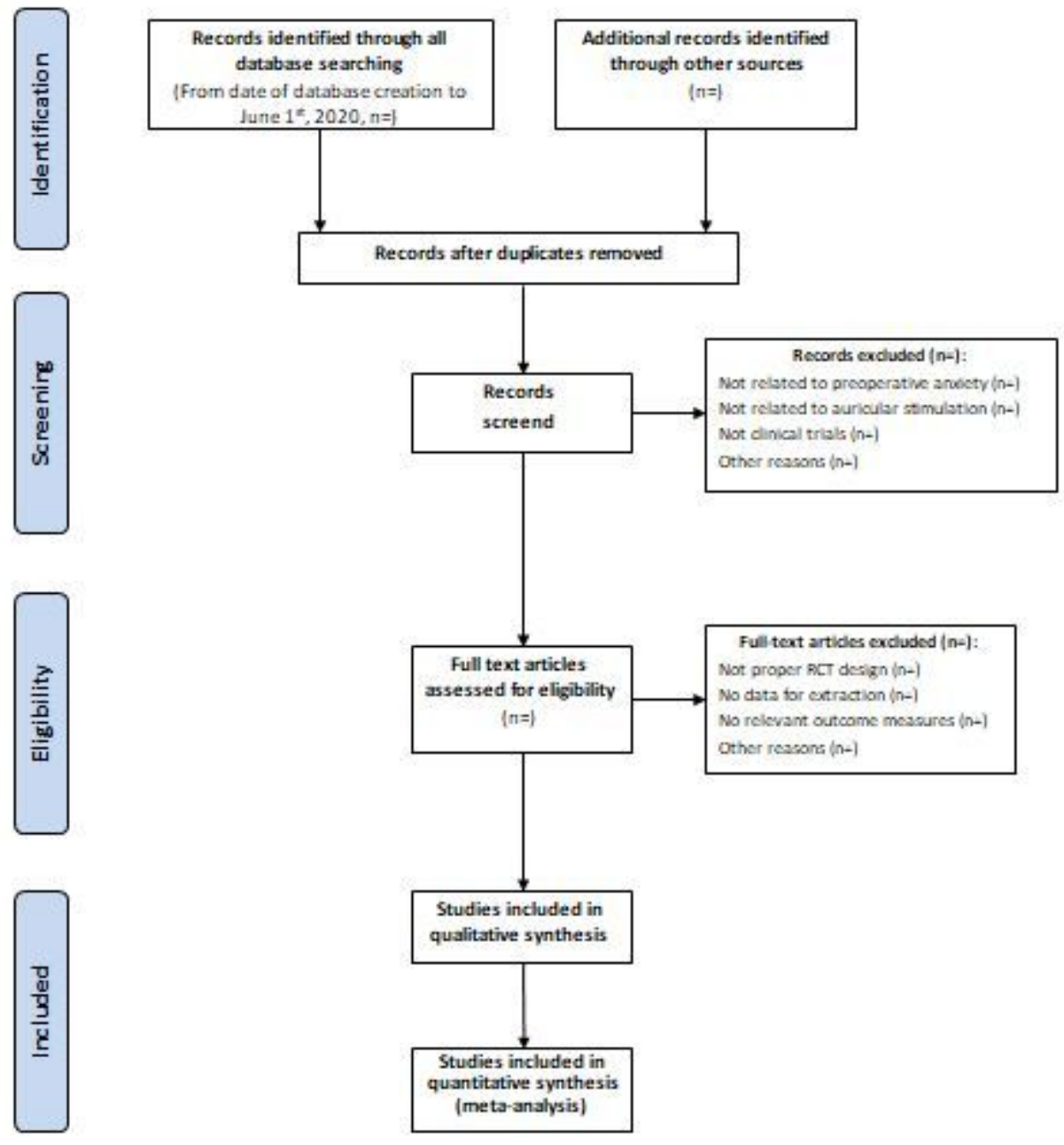

Figure 1

Flow diagram of details of data search and management

\section{Supplementary Files}

This is a list of supplementary files associated with this preprint. Click to download.

- DietzelSysrevAAforAnxietyprotocolPRISMA.docx 